\title{
The VOF method applied to the numerical simulation of a 2D liquid jet under gravity
}

\author{
G. Rocco ${ }^{1}$, G. Coppola ${ }^{2}$ \& L. de Luca ${ }^{1}$ \\ ${ }^{1}$ Dipartimento di Ingegneria Aerospaziale (DIAS), \\ Università degli Studi di Napoli "Federico II", Italia \\ ${ }^{2}$ Dipartmento di Energetica, Termofluidodinamica e Condizionamenti \\ ambientali (DETEC), Università degli Studi di Napoli "Federico II", \\ Italia
}

\begin{abstract}
Numerical simulations of a two-dimensional gravitational liquid sheet injected in another immiscible fluid are performed. The steady state of the liquid sheet has been calculated by solving the two-dimensional Navier-Stokes equations for variable density incompressible flows and the interface between the two fluids has been determined by using the Volume of Fluids method. The analysis takes into account viscous, inertial, gravitational and surface tension forces and different regimes of motion are identified according to the values of Reynolds and Stokes numbers. Velocity, pressure and shape of the sheet are investigated and the results are in well agreement with previous numerical and experimental results.
\end{abstract}

Keywords: VOF method, liquid jet, die-swell effect.

\section{Introduction}

The numerical simulation of free surface flows represents a very hard challenge due to the complex physical phenomena involved in the liquid-gas or liquidliquid interface dynamics, such as changes in topology (coalescence, break-up), instability, surface tension effects. A variety of engineering applications, concerning atomization, drops impact and liquid jets dynamics have motivated much research on this topic.

In particular, laminar liquid jets injected from a rectangular slot (liquid sheets) in an immiscible fluid have been studied extensively in the last years. 
The qualitative behaviour of a steady liquid sheet is well known. In the absence of gravity, at high Reynolds numbers the sheet contracts, whereas at low Reynolds numbers due to the sudden change of the boundary conditions on the viscous stresses, the sheet expands in the near field (die-swell effect, see Georgiou et al. [1]). Other important effects affecting the dynamics of the jet are surface tension and gravity. Among several contributions in the literature, two accurate references for the numerical simulation of the relevant flow field are represented by the works of de Luca and Costa [2] and Richards et al. [3]. In the former the numerical simulation of the liquid sheet is addressed by means of the so-called orthogonal Boundary-Fitted Coordinate Transformation (BFCT), while in the latter a Volume Of Fluids method (VOF) is applied to simulate a round liquid jet issuing into another liquid.

In the present paper a two-dimensional second order finite difference VOFcode, named MultiFluids, is developed to investigate the behaviour of a twodimensional liquid sheet issuing from a rectangular slot under the influence of the gravitational field. The aim of the present paper is to carry out wide spectrum numerical simulations concerned with various flow regimes. This application is still lacking in the VOF literature.

The remainder of this paper is organized as follows. In section 2 we give a brief description of the VOF method and we introduce the numerical peculiarities of MultiFluids code. In section 3 the liquid sheet problem is set out. Finally, in section 4 and 5 we report the results of the numerical simulations and some conclusions.

\section{Numerical method}

In this section we describe the method for tracking the interface between two fluids in a two-dimensional, nonreacting, incompressible flow. In recent years a number of methods have been developed for the solution of problems involving the motion of interfaces in multiphase flows. A possible classification of these methods is based on the type of grid used. From this point of view the various methods for interface simulation can be divided into two great classes. In the first class, the interface is treated as a boundary between two sub-domains. This approach requires a deformable grid in order to follow the motion of the interface. The principal disadvantage in the use of these methods is that the grid can undergo a considerable distortion. The second class of methods uses a predefined fixed grid that does not move with the interface. In this case, a specific advection scheme, able to convect the interface, is employed. These last schemes can be divided into explicit and implicit if the interface is explicitly or implicitly represented. Among the implicit methods there are the Level Set (LS) and VOF methods. The first one, introduced by Osher and Sethian [4], describes the interface as the zero level of a continuous scalar field, named level set function. This method consists updating the level set function at each time step by means of a transport equation. The principal advantage of this method, with respect to the VOF method, is that the approximation of the interface is globally continuous. Unfortunately, this method does not enforce the mass balance in a 
natural way. In the VOF method, introduced by Hirt and Nichols [5], the interface is represented by means of a Heaviside function, named volume of fraction function. In contrast to the LS method, the main advantage of this method is that it enforces the mass balance in a natural way, even for a relatively coarse grid. Furthermore, as in the LS method, no special treatments are required to model the topological changes of the interface or break-up phenomena. Unfortunately, this method uses a discontinuous function to describe the interface, thus the solution is affected by numerical diffusion and all topological information of the interface as, for example, the normal and the curvature, are approximated by smoothing the Heaviside function.

In the VOF methods, as well as in LS methods, the Navier-Stokes equations are used in a "one-fluid formulation" which requires solving only one set of equations for two immiscible fluids with different densities and viscosity. In order to identify the topology of the interface advected by the velocity field, a transport equation for the volume fraction function $f$ is coupled to the NavierStokes equations. This function is used to compute physical properties and local curvature of the interface. The equations of motion to be solved are:

$$
\begin{gathered}
\nabla \cdot \underline{V}=0 . \\
\rho \frac{\partial \underline{V}}{\partial t}+\rho \nabla \cdot \underline{V} \underline{V}=-\underline{\nabla p}+\nabla \cdot(2 \mu \underline{\underline{D}})+\underline{F}_{s}+\underline{F}_{b} . \\
\frac{\partial f}{\partial t}+\underline{V} \cdot \underline{\nabla f}=0 .
\end{gathered}
$$

where $\underline{F}_{b}$ is a body force, $\underline{\underline{D}}$ is the symmetric part of the strain tensor and $\underline{F}_{s}$ is the surface tension force. In eqns. (2), density and viscosity are not constant in all physical domain, but their value depends on the volume fraction function:

$$
\rho=\rho_{l} f+(1-f) \rho_{g}, \quad \mu=\mu_{l} f+(1-f) \mu_{g}
$$

where $\rho_{l}, \mu_{l}$ are the density and the viscosity of the liquid phase and $\rho_{g}, \mu_{g}$ are the density and the viscosity of the gas phase.

In last years many numerical methods have been proposed in order to model the effects of surface tension forces $\underline{F}_{s}$ in VOF codes. This is an important topic since in many free surface flows, such as fluids with high surface tension (or with surfactant), flows with convolute interfaces (high curvature) and pendant drops, surface tension forces are the dominant forces. The surface tension term in the Navier-Stokes equations creates the principal difficulties, since it is a singular term. In several implementations of the VOF method these difficulties can produce spurious currents and numerical instability (Renardy and Renardy [6]). In our code many of the most popular schemes for surface tension 
modelling have been tested, the numerical simulations here presented have been obtained by means of the Continuum Surface Force (CSF) model (Brackbill et al. [7]) because of its low computational cost and wide diffusion in many commercial and home-made numerical codes. In CSF model, the effects of surface tension forces are modelled by means of an explicit term in the NavierStokes equations:

$$
\underline{F}_{s}=\sigma k \delta_{s} \underline{n}_{s} .
$$

where $k$ is the curvature of the interface, $\delta_{s}$ is the Dirac function, $\sigma$ is the surface tension coefficient and $\underline{n}_{s}$ the normal unit vector to the interface. The curvature is calculated by evaluating the divergence of the normal unit vector to the interface and this vector is calculated by implementing finite differences of the volume fraction function smoothed with the filter defined in Lafaurie et al. [8]. Thus the surface tension effects are implemented in a simple way, by distributing it over neighbouring grid points.

In MultiFluids code, the Navier-Stokes equations are solved by means of the projection method on staggered uniform grid due to Chorin [9]. The special features of our code are: it is based on a explicit fourth order Runge-Kutta method; a second order lagrangian scheme for the volume fraction equation is used (Gueyffier et al. [10]); a CSF method is adopted in order to model the surface tension forces. A modified Neumann boundary condition is employed for the special treatment of the normal component of the velocity at the outflow boundary (Sander et al. [11]).

\section{Problem definition and numerical setup}

A stationary jet of water is ejected vertically in the gravitational field by means of a rectangular slot in an environment of quiescent air (figure 1(a)). The slot width is $2 b$, whereas along the $z$ direction the slot is infinite and the flow can be considered two-dimensional. The main dimensionless numbers governing the motion of the liquid sheet are Reynolds (Re), Stokes (St) and Capillary (Ca) numbers:

$$
\operatorname{Re}=\frac{2 u \rho_{l} b}{\mu_{l}}, \quad S t=\frac{4 \rho_{l} g b^{2}}{\mu_{l} u}, \quad C a=\frac{\mu_{l} u}{\sigma} .
$$

where $\rho_{l}, \mu_{l}$ are the density and the viscosity of the water, $u$ is the mean inflow velocity and $\sigma$ is the surface tension coefficient.

\subsection{Computational domain}

A sketch of computational domain is shown in Figure 1(b). The flow is assumed to be symmetric with respect to the slot symmetry plane ( $x-z$ plane in figure 1(a)), so the computational domain is a rectangle bounded by the symmetric plane of the slot at its west side, wall at north and open edges at east and south sides. The dimensions of the computational domain depends on the slot width. 
We assume that the dimension, in axial direction, is equal to $\mathrm{L}_{\mathrm{x}}=30 \mathrm{~b}$; whereas in the normal direction it is set to $\mathrm{L}_{\mathrm{y}}=10 \mathrm{~b}$. Numerical experiments carried out on different domain size confirm that these dimensions are sufficient to obtain a full-developed flow. The spatial resolution of computational domain was defined by a uniform grid spacing of $b / 10$ spatial step.

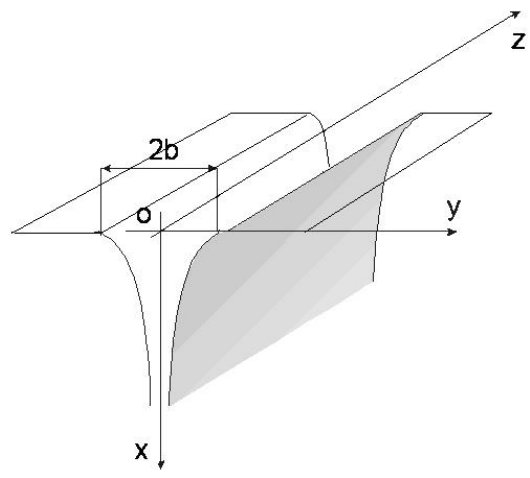

(a)

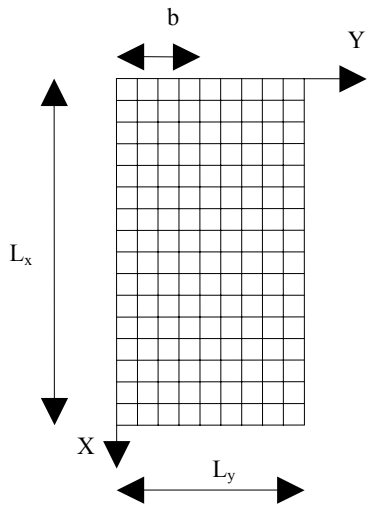

(b)

Figure 1: $\quad$ Schematic description of the (a) physical and (b) computational domain.

\subsection{Boundary and initial conditions}

Regarding the initial conditions, we use a rectangular form for the initial shape of the jet, whereas for the initial velocity conditions a Poiseuille profile is employed in the liquid phase and a zero velocity condition is imposed in the gaseous phase (quiescent ambient). For what it concerns boundary conditions, a Poiseuille velocity profile has been adopted for the inflow, whereas at the west edge of the computational domain a symmetry condition has been imposed. The treatment of the boundary conditions at east and south edges is more complex. In fact, in order to take into account the lateral inflow of the gas due to the typical entrainment effect occurring during the spread of the liquid jet, an open boundary condition is imposed on east edge of the computational domain. This condition consists in homogeneous Neumann conditions for both velocity components. At the outflow, in order to suppress numerical difficulties arising from the backflow, a modified Neumann boundary condition for the streamwise component of the velocity has been applied. The modification is based on the idea that if the streamwise component of the velocity, in the last computational cell, is positive, then classic homogeneous Neumann boundary condition is adopted. On contrary, if this velocity is negative (backflow), a Dirichlet boundary condition is adopted and the streamwise component of the velocity is set to zero. 


\section{Results}

In this section we present some results obtained by means of MultiFluids code. In order to give a better physical interpretation of the results we introduce a classification of different regimes of motion for various Reynolds and Stokes numbers. All the possible flow regimes are reported in table 1; for example, a liquid jet with high Reynolds and Stokes numbers is in Inertial-Gravitational regime, whereas a liquid jet, with low Reynolds and Stokes numbers is in Viscous regime. Capillary number can be seen as an additional governing parameter for each regimes identified in table 1 . Note that in the figures, the adopted Cartesian coordinate system $(x, y)$ denoting the axial and lateral coordinates are made dimensionless with respect to the half slot width.

Table 1: $\quad$ Classification of different flow regimes.

\begin{tabular}{c|ccc}
\hline & $\begin{array}{c}\text { Re }<<1 \\
\text { Creeping }\end{array}$ & $\operatorname{Re}=O(1)$ & $\begin{array}{c}\text { Re }>>1 \\
\text { Inertial }\end{array}$ \\
\hline $\begin{array}{c}\text { No gravity } \\
\text { regime }\end{array}$ & Viscous & $\begin{array}{c}\text { Viscous } \\
\text { Inertial }\end{array}$ & Inertial \\
$\mathrm{St}=\mathrm{O}(1)$ & $\begin{array}{c}\text { Viscous } \\
\text { Gravitational }\end{array}$ & $\begin{array}{c}\text { Viscous } \\
\text { Inertial } \\
\text { Gravitational }\end{array}$ & Inertial \\
$\begin{array}{c}\mathrm{St}>>1 \\
\begin{array}{c}\text { Gravitational } \\
\text { regime }\end{array}\end{array}$ & Gravitational & Gravitational & $\begin{array}{c}\text { Inertial } \\
\text { Gravitational }\end{array}$ \\
\hline & & & \\
\hline
\end{tabular}

\subsection{Vertically falling liquid sheet}

Figure 2 shows the solution during the transient evolutions of the liquid sheet without surface tension force at various time step computed at Reynolds number equal to 50 and Stokes number equal to 25 . Figure 3 refers to the same simulation of figure 2 and shows the steady shape of the computed interface (continuous line) together with the inviscid, or torricellian, solution (dotted line). This simulation refers to Inertia - Gravity regime for which the jet contracts and uncovers downstream the torricellian solution. The numerical solution appears more slender than the inviscid one in the near field according with literature results. Nevertheless, the contraction of the jet in the present simulations appears more pronounced than in de Luca and Costa [2]. Figure 4 shows the pressure field when the stationary conditions are reached. 


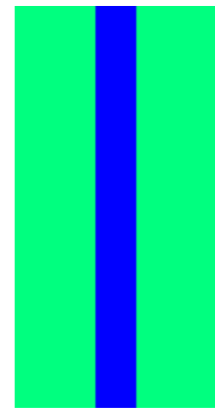

(a)

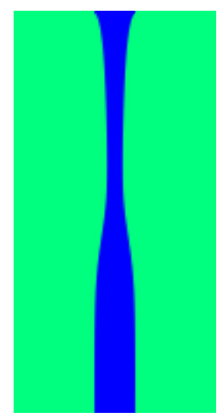

(d)

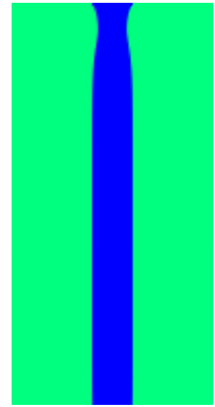

(b)

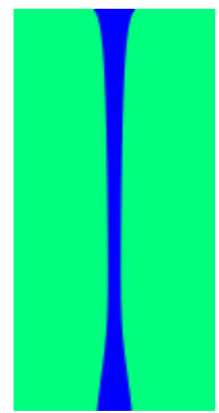

(e)

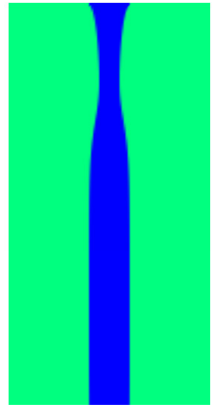

(c)

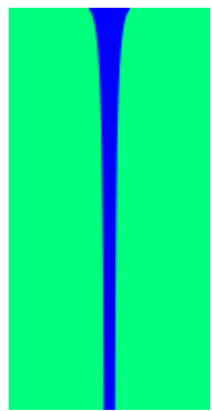

(f)

Figure 2: $\quad$ Transient evolution of the liquid sheet with $\mathrm{Ca}=\infty, \mathrm{Re}=50$ and $\mathrm{St}$ $=25 . \Delta \mathrm{t}=10^{-5} \mathrm{~s}$ (a) time step 1, (b) 10000, (c) 20000, (d) 30000, (e) 40000, (f) 50000 .

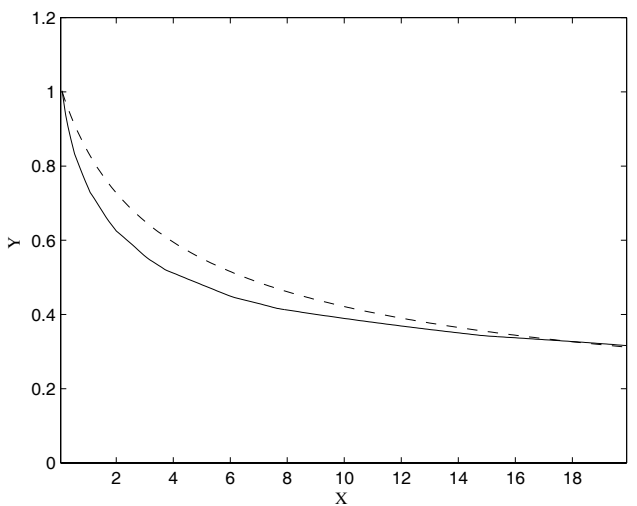

Figure 3: $\quad$ Stationary shape of the liquid sheet: $(-) \mathrm{Ca}=\infty, \mathrm{Re}=50$ and $\mathrm{St}=25$; (---) torricellian solution. 


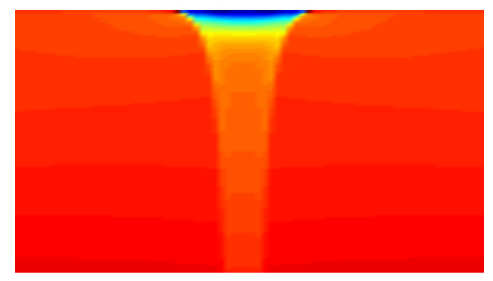

Figure 4: Pressure field in stationary condition at $\mathrm{Ca}=\infty, \mathrm{Re}=50$ and $\mathrm{St}=25$.

\subsection{No-gravity or low gravity jet swell problem}

A simulation of the jet behaviour in the Viscous - Inertial regime is represented in figure 5. In this regime, in the absence of gravity, the jet exhibits a very large swelling due to high viscous effects. According to Nickell et al.[12] the extrudate swell for this case approaches to 1.19. Figure 6 shows the classic dieswell phenomenon computed at $\mathrm{Re}=2$ and $\mathrm{St}=1$. This simulation refers to the Viscous-Inertial-Gravitational regime. In this regime the jet exhibits a remarkable swelling only in the entrance region. This behaviour is due to the combination of high viscous and low gravity local effects. Subsequently the gravitational effects produce a contraction of the jet that approaches the torricellian solution (dotted line). The comparison between figures 7(a) and 7(b) emphasizes the different behaviour between the jet in the gravitational and in the viscous regime and the role of the velocity fields on the deformed shape of the curtain. As we can see in figure 7(a), the velocity field causes a contraction of the jet in the Inertial regime, whereas in figure 7(b) a remarkable swelling due to velocity field is clearly evident.

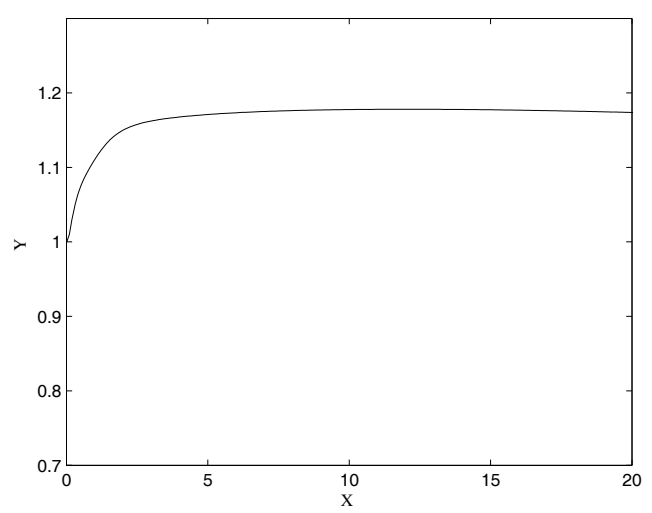

Figure 5: A detail of the liquid sheet at $\mathrm{Ca}=\infty, \mathrm{Re}=4$ and $\mathrm{St}=0$. 


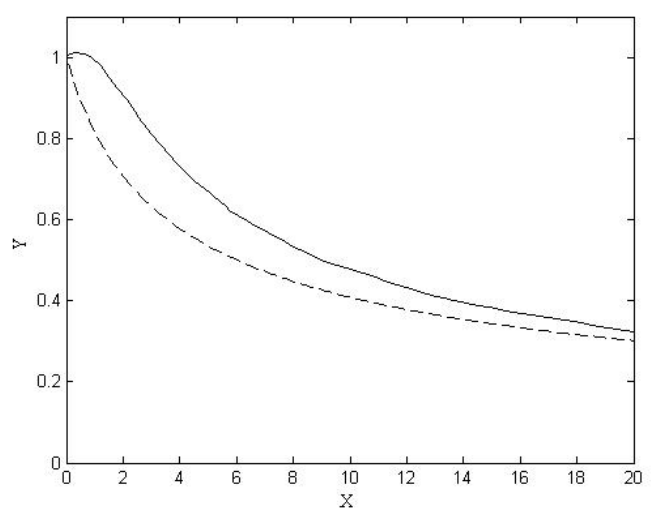

Figure 6: Stationary shape of the liquid sheet: $(-) \mathrm{Ca}=\infty, \mathrm{Re}=2$ and $\mathrm{St}=$ 1 ; (---) torricellian solution.

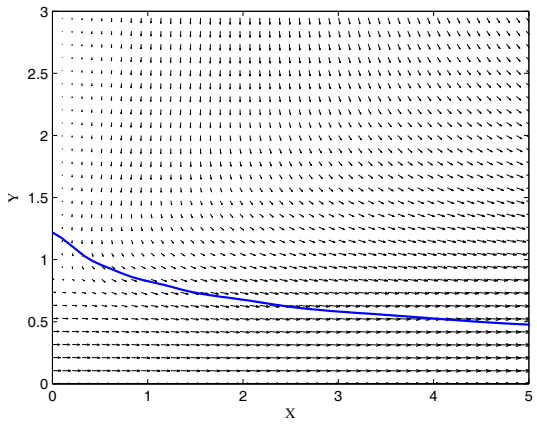

(a)

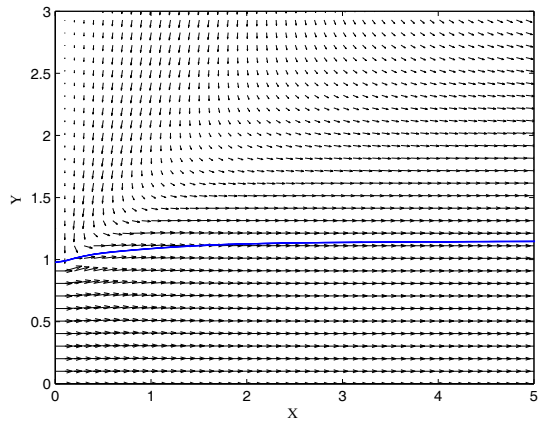

(b)

Figure 7: Velocity field and stationary shape of the liquid sheet $(-)$ at $\mathrm{Ca}=\infty$. (a) $\mathrm{Re}=50$ and $\mathrm{St}=25$, (b) $\mathrm{Re}=4$ and $\mathrm{St}=0$.

\subsection{Influence of the capillary number}

A comparison of the jet behaviour with and without surface tension effects is reported in figure 8 . In this figure we can see that the jet without surface tension forces (red line) appears more slender than the jet computed in the case including the effects of the surface tension force (black line). As a conclusion, the surface tension, in inertial gravity regime, tends to reduce the contraction of the sheet. 


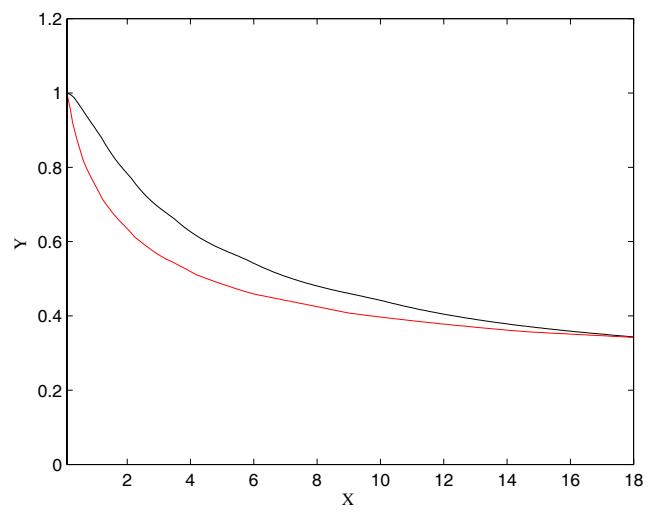

Figure 8: Influence of the capillary number on the stationary shape of the liquid sheet at $\mathrm{Re}=50$ and $\mathrm{St}=25:(-) \mathrm{Ca}=10^{-2} ;(-) \mathrm{Ca}=\infty$.

\section{Conclusion}

The stationary free surface flow of a two-dimensional plane liquid jet evolving in quiescent air in presence of gravity has been numerically determined by means of a VOF code. Inertia, viscous, gravity and surface tension are all included in the present model.

The results show that at low Stokes numbers (low gravity) the jet expands issuing from the slot and, subsequently, it contracts along the streamwise direction, whereas, at zero gravity, the jet expands and, if the Reynolds number is very low, the contraction is not present. The dynamics of die-swell phenomena is recovered. In the gravitational regime, the effect of the gravity is dominant and the jet contracts monotonically along the streamwise direction uncovering far downstream the inviscid solution.

The results are in good agreement with previous numerical simulations. This paper constitutes a preliminary step towards the numerical simulation of breakup phenomena, whose task is currently in progress.

\section{Reference}

[1] Georgiou G. C., Papanastasiou T. C. and Wilkes J. O., "Laminar Newtonian jets at high Reynolds number and high surface tension," AIChE Journal, v. 34, p. 1559-1562, 1988.

[2] de Luca L. and Costa M., "Two-dimensional flow of a liquid sheet under gravity," Computers \& Fluids, v. 24, p. 401-414, 1995.

[3] Richards J. R., Beris A. N., and Lenhoff A. M., "Steady laminar flow of liquid-liquid jets at high Reynolds numbers," Physics of Fluids, v. A 5, p. 1703-1717, 1993. 
[4] Osher S., Sethian J., "Fronts propagating with curvature-dependent speed: Algorithms based on Hamilton-Jacobi formulations," Journal of Computational Physics, v. 79, p. 12-49, 1988.

[5] Hirt C. W., Nichols B. D., "Volume of fluid (VOF) method for the dynamics of free boundaries," Journal of Computational Physics, v. 39, p. 201-235, 1981.

[6] Renardy Y., Renardy M., "PROST: A Parabolic Reconstruction of Surface Tension for the Volume-of-Fluid Method," Journal of Computational Physics, v. 183, p. 400-421, 2002.

[7] Brackbill J. U., Kote D. B., and Zemach C., "A continuum method for modelling surface tension," Journal of Computational Physics, v. 100, p. 335-354, 1992.

[8] Lafaurie B., Nardone C., Scardovelli R. and Zaleski S., "Modelling, merging and fragmentation in multiphase flow with SURFER," Journal of Computational Physics, v. 113, p. 134-147, 1994.

[9] Chorin A., "A numerical solution of the Navier-Stokes equations," Mathematics of Computation, v. 22, p. 745-762, 1968.

[10] Gueyffier D., Nadim A., Li J., Scardovelli R., Zaleski S., "Volume of fluid interface tracking with smoothed surface stress methods for threedimensional flows," Journal of Computational Physics, v. 152, p. 423-456, 1999.

[11] Sander W. and Weigand B., "Direct numerical simulation and analysis of instability enhancing parameters in liquid sheets at moderate Reynolds number," Physics of Fluids, v. 20 053301, p. 1-18, 2008.

[12] Nickell R. E., Tanner R. I. and Caswell B., "The Solution of Viscous Incompressible Flow Jet and Free-Surface Flows Using Finite-Element Methods," Journal of Fluid Mechanics, v. 65, p. 189-206, 1974. 